\title{
Dementia care in Ireland: what's the plan?
}

\author{
Henry O'Connell
}

Ir J Psych Med 2011; 28(3): 112-115

Dementia is rapidly emerging as one of the most significant and costly of all medical disorders in Ireland and worldwide. The impact of dementia on individual sufferers, their families and wider society is immense.

At present in Ireland, there are no clear pathways of referral for assessment, diagnosis and management of individuals with cognitive impairment/dementia. There is, effectively, no dementia strategy in operation. Instead, there is an ad hoc and disjointed approach to diagnosis and management of dementia at all stages, involving varying levels of input from primary care, public health, geriatric medicine, old age psychiatry, neurology and memory/cognitive disorder clinics, with no blueprint for how different services can and should interface.

Recent calls from the Alzheimer's Society of Ireland and the Dementia Services Information and Development Centre ${ }^{1}$ have again highlighted this significant gap in healthcare planning and the recent threat to the Nursing Home Support Scheme ('Fair Deal' scheme) ${ }^{2}$ has highlighted the need for dementia care to be prioritised in overall healthcare budgets.

Despite the emphasis in government health policy on the Primary Care Strategy ${ }^{3}$ in recent years, primary care does not routinely manage dementia diagnosis and management. Furthermore, there are no clear working arrangements linking primary care to specialist services, and interaction with public health and community care is equally ad hoc.

This paper is divided into four main sections. In the first section, a recent (2007) dementia strategy paper for Ireland is summarised. ${ }^{4}$ In the second section, the 1999 paper $A n$ Action Plan for Dementia is summarised. ${ }^{5}$ In the third section, key principles from Vision for Change are summarised. ${ }^{6}$ In the fourth section, two dementia strategy documents from the UK are summarised. ${ }^{7,8}$ In the final section, the key clinical challenges in managing dementia are outlined and some suggestions are proposed for the effective management of dementia care in the years to come.

Implementing Policy for Dementia care in Ireland The Time for Action is Now ${ }^{4}$

In this paper, Professor Eamon O'Shea (for the Alzheimer Society of Ireland) provides a timely review of dementia care in Ireland and highlights again the need for increased funding and coordination of services directed towards people with dementia, for the purposes of early diagnosis, treatment and care, both in the community and in residential settings.

Henry O'Connell, Consultant Psychiatrist, Psychiatry of Later Life, Laois-Offaly Mental Health Services, An Triu Aois Day Hospital, Block Road, Portlaoise, Co Laois and Adjunct Senior Clinical Lecturer at the University of Limerick Graduate Entry Medical School, Ireland.

SUBMITTED: JANUARY 24, 2011. ACCEPTED: AUGUST 25, 2011.

\begin{tabular}{|lc|}
\hline Table 1: Projected prevalence and incidence of dementia in lreland \\
\hline Year & Persons with dementia (1'Shea, 2007) \\
2006 & 37,746 \\
2011 & 42,441 \\
2016 & 49,153 \\
2021 & 58,044 \\
2026 & 70,115 \\
2031 & 85,847 \\
2036 & 103,998 \\
\hline
\end{tabular}

The first three sections of this paper provide an introduction and background information on the clinical presentation of dementia.

The fourth section relates to the projected prevalence and incidence of dementia in Ireland (see Table 1).

In the subsequent sections, O'Shea summarises the impact on families of dementia care. For example, it is estimated that 50,000 carers in Ireland look after someone with one of six specified symptoms of dementia and that the median daily provision of care provided by these carers to people with dementia is 10 hours.

The economic impact of dementia is also summarised in this paper, along with some projected costings for dementia care. O'Shea highlights that the original costs for the Action Plan for Dementia were modest, ${ }^{5}$ requiring $€ 20$ million per year over a three year period, amounting to an annual grant of just over $€ 500$ per person with dementia. O'Shea estimates that the total cost for implementing the Action Plan for $D_{\text {Dementia }}{ }^{5}$ in 2007 would be $€ 73$ million, made over a three year period, to bridge the gap between need and existing provision for people with dementia.

O'Shea estimates the 2006 baseline estimate for dementia care in Ireland at just under $€ 400$ million, a substantial sum but only a tiny figure in comparison to the many tens of billions that have been used to 'bail out' the Irish banking system over the past two years. Of this $€ 400$ million, O'Shea estimates that $6 \%$ is attributable to community care services, with nearly one third of the burden of care attributable to residential care.

The annual per capita cost of dementia care in Ireland in $2004^{9}$ was estimated at just under $€ 10,000$, somewhere intermediate between the countries with the highest cost of care (Finland: $€ 19,458$ ) and the lowest (France: $€ 5,981$ ).

O'Shea also estimates that in 2007 there were 1,300 highly dependent people with dementia living at home and, at a cost per home care package costed $€ 350$ per week, then 


\section{Table 2: Gore principles in the 'Action Plan for Dementia}

1. Person centred models of care which respect the preferences and rights of the person with dementia

2. Comprehensive provision

3. Bias towards home care solutions

4. Care requirements to determine funding

5. Needs-based provision

6. Evidence-based practice linked to national quality and outcome targets

\section{Table 3: Main targets outlined in 'Action Plan for Dementia'}

1. Increased public awareness about dementia

2. Increase in early diagnosis through enhanced training and education for primary care workers, especially for general practitioners

3. Development of a care management model of integrated care

4. Expansion of dedicated community-based services, eg. day-care services, occupational therapists, community psychiatric nurses

5. Expansion of dedicated old age psychiatry services

6. Development of new and expanded psychosocial approaches to compliment existing medical and neurological models of service delivery in the community and residential care units

7. Development of small-scale, appropriately assigned, special residential care units

8. Development of new services for people with early onset dementia including people with Down's syndrome

their needs could be met at an annual cost of $€ 23$ million.

In the next section, current policy is summarised and an outline is given of future strategies and priorities. O'Shea reverts back to the Action Plan for Dementia ${ }^{5}$ and highlights again the six core principles and the eight main targets involved. These are listed in the next section of this paper.

O'Shea goes on to highlight the fact that, while the government's 2001 Health Strategy: Quality and Fairness: A Health System For You ${ }^{10}$ accepted the general thrust of the Action Plan for Dementia ${ }^{5}$ and committed to its implementation over a seven year period, progress has been slow in implementing this.

Future strategies and priorities are then described. O'Shea highlights that dementia should be accorded special status as an illness, that it should be made a national health priority and that it should have a national strategy of its own. The national prioritisation of dementia should encompass four key strategic elements, each of which have their own origins in the Action Plan for Dementia:

1. Early intervention, education and training

2. Enhanced community-based services for people with dementia and their carers

3. Co-ordination and integration of multi-disciplinary care provision

4. Quality residential care to be centred on the person with dementia.

\section{Conclusions}

In conclusion, O'Shea urges that dementia should be
Table 4: Key recommendations from 'Action Plan for Dementia's regarding old age psychiatry services

- The provision of one consultant in old age psychiatry per 10,000 people age 65 years and over, each being provided with an appropriate multi-disciplinary team and psychiatric facilities including a day hospital and acute and longstay psychiatric beds together with good access to non-psychiatric day and residential care.

- The development of day hospitals within each psychiatry of old age service for people with dementia who have associated behavioural problems or psychiatric symptoms.

- The recommendation that there be an adoption of 1 in 4,500 elderly population for community psychiatric nursing services within the framework of an expanded old age psychiatry service.

- Close cooperation was encouraged between geriatric medicine and psychiatry of old age services

made a national health priority, beginning with the full and accelerated implementation of the Action Plan for Dementia. ${ }^{5} \mathrm{He}$ highlights the need to have a strategy that relates to people at all stages of dementia and their carers. Emphasis should be placed on primary care and community care solutions to the problems that currently exist in this area. Families are acknowledged as providing the bulk of care for people with dementia and they should be afforded the appropriate support.

\section{Comments}

While some costs of dementia care are provided, more emphasis should be placed on the detailed and exact costing of implementing the different strands of a national dementia strategy, and the mechanics of how such a strategy can be implemented, using existing structures. Such costing also now needs to be put in the context of the dramatic economic downturn in this country since 2008 .

A notable omission from O'Shea's paper is the lack of any reference to $A$ Vision for Change, ${ }^{6}$ although it is likely that O'Shea's paper was largely written before the publication of that document. Ideally, the data and recommendations from O'Shea's 2007 paper should be integrated where appropriate with the principles of $A$ Vision for Change.

\section{An Action Plan for Dementia ${ }^{5}$}

This important document, published in 1999, presented the key points in relation to the existing level of service provision for dementia and proposed future targets and concluded with 33 recommendations. The core principles and main targets of this document are outlined above in Tables 2 and 3.

Regarding old age psychiatry (see Table 4), this paper reflected the policy of the Irish Division of the Royal College of Psychiatrists, as it existed at the time, and this policy has since been superseded by the principles contained within Vision for Change. ${ }^{6}$

However, the principle of primary and community care services dealing with the majority of dementia cases, with old age psychiatry becoming involved when there are problems with behavioural or psychiatric symptoms, is in keeping with the general principles of Vision for Change.

The principles contained in this document are sound and commendable, covering dementia care from early intervention through to residential care and highlighting the importance of 
needs-based provision. Partly because of the lack of implementation of the recommendations of this report, O'Shea produced the 2007 document for the Alzheimer Society of Ireland. ${ }^{4}$

\section{Vision for Change ${ }^{6}$}

Many of the general principles contained within Vision for Change ${ }^{6}$ can be related directly or indirectly to dementia care. These principles include early intervention, accessibility of services, a population health approach and use of the biopsychosocial model.

The framework for a comprehensive mental health policy, with community support forming the base of the triangle and primary care and mental health services above this can also be related to dementia care.

Other general principles that can be related to dementia care include the importance of involving service users and carers in care planning and the need for a framework for interdepartmental co-operation in the development of crosscutting health and social policy to be put in place.

As a further general principle, more research and information is required on the prevalence of dementia, to effectively plan primary care services and the interface between primary care and specialist mental health services.

Specifically regarding dementia, Vision for Change ${ }^{6}$ states that any person, aged 65 years or over, with secondary behavioural and affective problems arising from experience of dementia, has the right to be cared for by mental health services for older people.

Vision for Change $^{6}$ also recommends that primary health care teams should play a major role in assessment and screening for mental illness in older people and should work in a coordinated and integrated manner with the specialist teams to provide high-quality care, particularly care that is home-based. This principle is particularly applicable to dementia diagnosis and management.

Vision for Change ${ }^{6}$ recommends appropriate linkage with voluntary agencies and highlights that carers and families should receive appropriate recognition and support including education, respite and crisis response when required.

Finally, Vision for Change ${ }^{6}$ recommends that older people with mental health problems should have access to nursing homes on the same basis as the rest of the population.

\section{Comments}

While many of the principles of Vision for Change ${ }^{6}$ are commendable, the document is short on specific recommendations in many areas, including the whole area of dementia care.

Only the general principles contained within Vision for Change ${ }^{6}$ are included in the above paragraphs, and it must be borne in mind that there are also significant concerns within old age psychiatry about this document, especially considering the limited input from old age psychiatry in its development.

In particular, old age psychiatry consultation-liaison services are not acknowledged within Vision for Change ${ }^{6}$. This one area alone is a significant component of an overall dementia strategy, considering the high levels of delirium and dementia that are managed by old age psychiatry consultation-liaison services on an ongoing basis.

\section{Table 5: Strategy objectives for 'Living well with dementia: A National}

Dementia Strategy', Department of Health, UK ${ }^{7}$

Objective 1: Improving public and professional awareness and understanding of dementia

Objective 2: Good quality early diagnosis and intervention for all

Objective 3: Good quality information for those who diagnose dementia and their carers

Objective 4: Enabling easy access to care, support and advice following diagnosis

Objective 5: Development of structured peer support and learning networks.

Objective 6: Improved community personal support services

Objective 7: Implementing the Carers' Strategy

Objective 8: Improved quality of care for people with dementia in general hospitals

Objective 9: Improved intermediate care for people with dementia

Objective 10: Considering the potential for housing support, housing-related services and telecare to support people with dementia and their carers

Objective 11: Living well with dementia in care homes.

Objective 12: Improved end of life care for people with dementia

Objective 13: An informed and effective workforce for people with dementia

Objective 14: A joint commissioning strategy for dementia

Objective 15: Improved assessment and regulation of health and care services and of how systems are working for people with dementia and their carers

Objective 16: A clear picture of research evidence and needs

Objective 17: Effective national and regional support for implementation of the strategy.

\section{Dementia Strategy in the UK}

The aim of the UK strategy for England ${ }^{7}$ is to ensure that significant improvements are made to dementia services in three key areas: improved awareness, earlier diagnosis and intervention, and a higher quality of care.

A consultation process was conducted, involving over 50 stakeholder groups, attended by over 4,000 individuals, and approximately 600 responses to the consultation document were received.

The final document Living well with dementia: A National Dementia Strategy outlines 17 key strategy objectives relating to the development of a dementia strategy for England. These objectives are outlined above in Table 5.

While there are clearly likely to be many different national variations between Ireland and England, and the UK document is broader in scope than the Irish documents referred to in this paper, the principles of the UK document are again similar to those outlined in the Irish documents.

Overall, this is a comprehensive document, covering the different clinical contexts in which dementia is managed and acknowledging the central importance of carers (Objective 17). Objective eight relates to management of dementia in general hospitals, again highlighting the importance of old 


\section{Table 6: DHSSPS dementia strategy (Northern Ireland)}

- Prevention - there is some scope to prevent or delay the onset of dementia-mainly through a healthy lifestyle approach and reduction in cardiovascular risk factors

- Raising awareness and addressing stigma associated with the condition

- Access to early diagnosis, enhancing existing memory services to agreed standards to provide assessment, diagnosis, information and support

- Staged approach to care and support as the condition progresses, with the aim of maintaining daily living and independence as far as possible

- Improving staff awareness and skills to respond appropriately to people's needs;

- Redesign of services to provide care and support, as far as possible, in people's own homes and avoid admission to hospital or care home

age psychiatry consultation-liaison work in an overall dementia strategy.

The Department of Health, Social Services and Public Safety in Northern Ireland (DHSSPS) has also published a draft strategy on dementia services, Improving Dementia Services in Northern Ireland-A Regional Strategy. ${ }^{8}$ The key messages in the Northern Ireland strategy are outlined above in Table 6.

Again, this is a comprehensive and commendable document and the particular emphasis on dementia prevention raises a very important public health concern. Any future dementia strategies should take into account the importance of dementia prevention, from lifestyle and dietary habits through to management of cardiovascular risk factors.

\section{Conclusions}

The documents summarised in this paper highlight the varied nature and the huge extent of clinical and social problems posed by dementia, the current lack of any coherent, working strategy for the assessment, diagnosis and treatment of dementia and the urgent need for the development and expansion of a working dementia strategy for Ireland.

The lack of a coherent and functioning dementia strategy in Ireland has a direct impact on individuals with cognitive impairment/dementia and their families and carers, who have no clear pathways for accessing assessment, diagnosis and treatment of what is a profoundly life-changing and ultimately terminal disease.

The principles and recommendations outlined in the documents summarised in this paper are all reasonable expectations of a modern health service. However, many of the principles and recommendations are not in place and, when they are, they are implemented in an ad hoc and disjointed fashion, often with significant differences in the level of service provision between and even within the different geographical divisions of the Health Service Executive.

The impact of dementia on individuals, their carers, healthcare services and society generally is immense and is likely to rise significantly in future years, with projections for vastly increasing numbers of dementia sufferers and increased expectations for early diagnosis and more timely interventions.

Healthcare services in Ireland are already straining to manage the many problems associated with dementia care, in the community, the acute hospital setting and in respite and long-term care facilities.

While we have reliable information on the levels of dementia in Ireland and a number of useful policy documents, as outlined in this paper, there is now a need for a new dementia strategy for Ireland, taking into account the future projections for dementia prevalence and our changed economic climate.

Such a strategy should be comprehensive and multidisciplinary in scope, covering dementia at all stages of presentation and severity along with prevention and early intervention measures.

A new strategy should be placed in the context of current services as they exist throughout the country and appropriate costings for implementation should also be included. National standards should be defined, in the prevention, diagnosis and management of all stages of dementia, including palliative care. The key stakeholders to involve in formulating a new dementia strategy for Ireland would include carers, family members and dementia sufferers themselves, along with the key disciplines of primary care, public health, old age psychiatry, geriatric medicine, neurology and memory/cognitive disorders clinics.

\section{Declaration of Interest: None}

References

1. Cahill S. Developing a national dementia strategy for Ireland. Int J Geriatr Psychiatry 2010; 25(9): 912-6.

2. Nursing Home Support Scheme. www.oireachtas.ie/documents/bills28/acts/2009/ a1509.pdf

3. Primary Care - A New Direction Department of Health and Children, 2001. www. dohc.ie/publications

4. O'Shea E. Implementing Policy for Dementia Care in Ireland - The Time for Action is Now, 2007. The Alzheimer Society of Ireland.

5. O'Shea E and O'Reilly S. An Action Plan for Dementia. Report no. 54, 1999. National Council on Ageing and Older People.

6. A Vision for Change. 2006. The Stationery Office. www.dohc.ie/publications/vision for_change.html

7. Living well with dementia: A National Dementia Strategy, 2009. Department of Health 7. Living
(UK).

8. Consultation on Improving Dementia Services in Northern Ireland-A Regional Strategy 8. Consultation on Improving D

9. Jonsson $L$ and Berr C. Cost of Dementia in Europe. European Journal of Neurology 9. Jonsson $L$ and

$2005 ; 12: 50-53$.
10. Department of Health and Children, 2001. Quality and Fairness: A Health System for You. Dublin. Stationery Office. 